\section{A Geometric Characterization of Feedforward Forms}

\author{
A. Astolfi and G. Kaliora
}

\begin{abstract}
This note addresses the problem of (local) coordinates and feedback equivalence of single-input affine nonlinear systems to feedforward forms. In particular, using the notions of invariant and controlled invariant distributions we provide necessary and sufficient conditions for a general affine in control nonlinear system to be (locally) coordinates or feedback equivalent to a system in (strict) feedforward form. For illustration, the general theory is applied to a simple four-dimensional system describing the dynamics of a food-chain.
\end{abstract}

Index Terms-Coordinates equivalence, feedback equivalence, feedforward systems, invariant distributions.

\section{INTRODUCTION}

Following the seminal work [24] of Teel on stabilization of nonlinear systems, a new class of systems, denominated feedforward systems have attracted the attention of the nonlinear control community. Feedforward systems are in general not feedback linearizable and occur naturally in the model of simple physical systems, e.g., the cart and pendulum system, the ball and beam (with friction). Therefore, they have been regarded as an interesting class of truly nonlinear systems, for which methods such as feedback linearization or backstepping cannot be applied. Most of the attention of the researchers has been devoted to the stabilization problem and several semiglobal and global stabilization results have been derived, either via full state feedback or via measurement feedback; see, e.g., [10], [8], [17], [24], [18], [16], [4], [22], [7], and the references therein.

Most of the aforementioned works start from the assumption that the system to be controlled is already in feedforward form. As a result, little attention has been devoted to the problem of the intrinsic characterization of feedforward systems, i.e., to the problem of deciding when a given nonlinear system can be (locally or globally) transformed, via a coordinates or a feedback transformation, into a feedforward system. Notable exceptions are the results in [24, App. 1], [23], and [21]. In [24, App. 1], some sufficient conditions are proposed for the problem of feedback equivalence to strict feedforward forms. However, it must be noted that the (sufficient) conditions in [24, App. 1] rely on a special structure of the system to be transformed and on a special form for the transformed system. In particular, it is required that the transformed system is controllable in the first approximation and moreover that it can be decomposed into a feedback linearizable term and a perturbation term. The conditions for equivalence to strict feedforward form are then phrased in terms of the perturbation term (see also [15] for a similar structure, where the perturbation term is nonaffine in control). On the other hand, in [23] a single-input-single-output affine system is first approximated by its Taylor series expansion and then the problem of transforming it in a strict feedforward form is broken down to successively transforming vector fields that collect homogenous polynomials of degree $m$, for all $m \geq 2$ (see also [12] and [11]). This is achieved by a series of coordinates and feedback transformations each of which is again defined with homogeneous polynomials of degree $m$ and leaves

Manuscript received March 22, 2000; revised December 17, 2002, November 6, 2003, and January 12, 2005. Recommended by Associate Editor J. M. A. Scherpen. The work of A. Astolfi was supported in part by the Leverhulme Trust.

The authors are with the Electrical Engineering Department, Imperial College London, London SW7 2AZ, U.K. (e-mail: a.astolfi@ic.ac.uk; g.kaliora@ic.ac.uk).

Digital Object Identifier 10.1109/TAC.2005.851446 invariant the part of the system of homogeneous degree less than $m$. Necessary and sufficient conditions that guarantee the applicability of the method at each step are given, while finally the transformation is done up to a certain degree of accuracy, i.e., after $k$ successive homogeneous transformations, the equivalent representation of the system contains a vector field, that collects all nonlinear terms of degree larger than $k$, that is not in strict feedforward form. Finally, it is interesting to stress the following issues. First, feedforward systems are in general nonaffine in the control, i.e., the control variable does not enter linearly in the differential equations describing the systems, and this makes the equivalence problem more difficult. Second, there is not a unique definition of feedforward systems, i.e., the denomination feedforward systems is used to indicate different objects [8], [17], [24]. Moreover, a system is in feedforward form if, besides the structural properties, it fulfills also some controllability and observability like assumptions, that are used to obtain globally stabilizing control laws. We overcome these difficulties in the following way. First, we focus our attention on control affine systems, as the more general case of nonaffine systems can be dealt with using the idea of dynamic extension, which has already been used in the framework of feedback linearization of nonaffine systems; see, e.g., [19, Th. 6.12] (see also [14] where results on the stabilization of nonaffine triangular systems are presented). Second, we consider only the simplest possible description of feedforward systems and we do not take into consideration controllability and detectability issues.

The note studies necessary and sufficient conditions for systems equivalence to (strict) feedforward forms. This problem has been studied in [3], however, therein some of the conditions for the geometric characterization of strict feedforward forms are incorrect. The equivalence to feedforward forms is translated to the existence of invariant and control invariant distributions, and/or smooth functions that satisfy some geometric conditions, that are, however, coordinates free. Although some easy-to-check necessary conditions are also given, it has to be understood that the main results of the note consist of necessary and sufficient conditions that are only conceptual, i.e., they rely on the existence of solutions of certain partial differential equations, hence, in practice, they may not be easy to use. It must be noted however, that the existence of equivalent conditions for feedforward forms that are phrased in terms of the system vector fields (as in the case of feedback linearizability) is still an open issue. Compared to the work of [23] the results presented here are less constructive, however, as they are phrased for the system in its original nonlinear form, rather than for a polynomial approximation, they are likely to provide a more intuitive geometric interpretation. Moreover, contrary to what is most common in the literature of equivalence to feedforward forms, in this note, coordinates equivalence is examined in depth. Coordinates equivalence is the only option for transformation when autonomous systems, i.e., systems with no inputs, are considered. Note that the trajectories of a system written in a strict feedforward form can be explicitly computed. Besides that, even when controlled systems are considered, a feedback transformation may not always be possible, if, e.g., the control signal has to comply with magnitude constraints. Coordinates equivalence to strict feedforward forms, moreover, is strongly related to the existence of symmetry groups, as explained in [13, Sec. II.3]. Finally, results regarding coordinates equivalence to feedforward forms for discrete time nonlinear systems have been presented in [1] and [2].

\section{DEFinitions, BASIC FACTS, AND PROBlem Formulation}

In this section, we recall some definitions and facts from geometric control theory (see [6] and [19] for further details), we define two 
special classes of feedforward systems for which a geometric characterization will be given, and we state formally the problems under investigation.

Definition 1: A distribution $\Delta$ is said to be invariant under the vector field $f$ if $\tau \in \Delta \Rightarrow[f, \tau] \in \Delta$, i.e., the Lie bracket of $f$ with every vector field $\tau$ in $\Delta$ is a vector field in $\Delta$.

Definition 2: Consider a single-input system described by equations of the form

$$
\dot{x}=f(x)+g(x) u
$$

with $x \in U \subset I R^{n}$. A distribution $\Delta$ is said to be controlled invariant on $U$ if there exists a feedback control law described by equations of the form $u=\alpha(x)+\beta(x) v$ such that $[f+g \alpha, \Delta] \subset \Delta$ and $[g \beta, \Delta] \subset \Delta$ for all $x$ in $U$. A distribution $\Delta$ is said to be locally controlled invariant if for each $x \in U$ there exists a neighborhood $U^{0}$ of $x$ with the property that $\Delta$ is controlled invariant on $U^{0}$.

The notion of local controlled invariance can be easily tested in geometric terms, as expressed in [6, Lemma 6.2.1]. As already observed, there is no unique definition of feedforward systems. We focus on two special classes of systems, referred to as feedforward forms and strict feedforward forms.

Definition 3: The single-input affine nonlinear system (1), with state $x \in U \subset I R^{n}$, is said to be in feedforward form if the vector fields $f(x)$ and $g(x)$ are described by equations of the form

$$
f(x)=\left[\begin{array}{c}
f_{1}\left(x_{1}, \ldots, x_{n}\right) \\
f_{2}\left(x_{2}, \ldots, x_{n}\right) \\
\vdots \\
f_{n}\left(x_{n}\right)
\end{array}\right] \quad g(x)=\left[\begin{array}{c}
g_{1}\left(x_{1}, \ldots, x_{n}\right) \\
g_{2}\left(x_{2}, \ldots, x_{n}\right) \\
\vdots \\
g_{n}\left(x_{n}\right)
\end{array}\right] .
$$

Definition 4: The single-input affine nonlinear system (1), with state $x \in U \subset I R^{n}$, is said to be in strict feedforward form if the vector fields $f(x)$ and $g(x)$ are described by equations of the form

$$
f(x)=\left[\begin{array}{c}
f_{1}\left(x_{2}, \ldots, x_{n}\right) \\
f_{2}\left(x_{3}, \ldots, x_{n}\right) \\
\vdots \\
0
\end{array}\right] \quad g(x)=\left[\begin{array}{c}
g_{1}\left(x_{2}, \ldots, x_{n}\right) \\
g_{2}\left(x_{3}, \ldots, x_{n}\right) \\
\vdots \\
c
\end{array}\right]
$$

with $c \in I R$. Note that, without loss of generality, we can assume $c=1 .^{1}$

Remark 1: In the last definition, we could have set the last entry of the vector field $f(x)=f_{n} \in I R$ and the last entry of the vector field $g(x)=g_{n} \in I R$. Note however, that, if $g_{n} \neq 0$ it is always possible to redefine the input $u$ to obtain the form given in Definition 4. If $g_{n}=0$ and $f_{n} \neq 0$ then the system does not possess any equilibrium. Although this situation may be interesting from a theoretical point of view, it does not have any practical interest. Finally, if $g_{n}=0$ and $f_{n}=0$ then $x_{n}=x_{n}(0)$, and it is possible to consider reduced vector fields.

We are now ready to give a precise formulation of the problem addressed in this note.

Problem 1: (Coordinates equivalence problem) Given a single-input nonlinear system described by equations of the form (1), with $x \in U \subset I R^{n}$, and a point $x^{0} \in U$ find (if possible) a neighborhood $U^{0}$ of $x^{0}$ and a coordinates transformation $z=\Phi(x)$, defined on $U^{0}$, such that, in the new coordinates $z$, the system is in (strict) feedforward form.

Problem 2: (Feedback equivalence problem) Given a single-input nonlinear system described by equations of the form (1), with $x \in$

${ }^{1}$ See also [23] where the function $f_{n-1}\left(x_{n}\right)$ is, in addition, required to be linear and $g(x)=\left[\begin{array}{lllll}0 & 0 & \cdots & 0 & 1\end{array}\right]^{\prime}$.
$U \subset I R^{n}$, and a point $x^{0} \in U$ find (if possible) a neighborhood $U^{0}$ of $x^{0}$, a coordinates transformation $z=\Phi(x)$, defined on $U^{0}$, and a state feedback control law $u=\alpha(x)+\beta(x) v$ such that, in the new coordinates $z$, the closed-loop system $\dot{x}=f(x)+g(x) \alpha(x)+g(x) \beta(x) v$ is in (strict) feedforward form.

\section{MAIN RESULTS}

\section{A. Coordinates Equivalence}

The problem of coordinates equivalence to feedforward forms can be addressed and solved by a combination of the results of $[19$, Lemma 6.4] and [19, Th. 3.49] or [6, Lemma 1.6.1]. We state the following without proof.

Proposition 1: System (1) is locally coordinate equivalent to a system in feedforward form if and only if there exists a nested sequence of distributions

$$
\Delta_{1} \subset \Delta_{2} \subset \cdots \subset \Delta_{n-1}
$$

with $\Delta_{i} i$-dimensional and involutive, such that, for $i=1, \ldots, n-1$

$$
\left[f, \Delta_{i}\right] \subset \Delta_{i} \text { and }\left[g, \Delta_{i}\right] \subset \Delta_{i}
$$

The conditions in Proposition 1 can be strengthened to obtain a characterization of strict feedforward systems, as illustrated in the following statement.

Proposition 2: System (1) is locally coordinate equivalent to a system in strict feedforward form if and only if there exist a nested sequence of distributions as in expression (2), with $\Delta_{i} i$-dimensional and involutive and $n$ real functions $\lambda_{1}(x), \lambda_{2}(x), \ldots, \lambda_{n}(x)$, with $\lambda_{i}\left(x^{0}\right)=0$ and $d \lambda_{i}\left(x^{0}\right) \neq 0,{ }^{2}$ such that

$$
\begin{aligned}
& \Delta_{1}^{\perp} \not \supset d \lambda_{1} \in T^{*}\left(I R^{n}\right) \\
& \Delta_{2}^{\perp} \not \supset d \lambda_{2} \in \Delta_{1}^{\perp} \\
& \vdots \\
& \Delta_{n-1}^{\perp} \not \supset d \lambda_{n-1} \in \Delta_{n-2}^{\perp} \\
& 0 \neq d \lambda_{n} \in \Delta_{n-1}^{\perp}
\end{aligned}
$$

and

$$
\begin{array}{cc}
d L_{f} \lambda_{1} \in \Delta_{1}^{\perp} & d L_{g} \lambda_{1} \in \Delta_{1}^{\perp} \\
d L_{f} \lambda_{2} \in \Delta_{2}^{\perp} & d L_{g} \lambda_{2} \in \Delta_{2}^{\perp} \\
\vdots & \vdots \\
d L_{f} \lambda_{n-1} \in \Delta_{n-1}^{\perp} & d L_{g} \lambda_{n-1} \in \Delta_{n-1}^{\perp} \\
d L_{f} \lambda_{n}=0 & d L_{g} \lambda_{n}=0
\end{array}
$$

where $T^{*}\left(I R^{n}\right)$ denotes the cotangent bundle of $I R^{n}$.

Proof: (Only If) Assume there exists a local coordinates transformation $y=\Phi(x)$ such that the transformed system with state $y$ is in strict feedforward form. Then, simple computations show that the distributions $\Delta_{i}=\operatorname{span}\left\{\left((\partial) /\left(\partial y_{1}\right)\right), \ldots,\left((\partial) /\left(\partial y_{i}\right)\right)\right\}$ are $i$-dimensional and involutive. Moreover, the functions $\lambda_{1}(y)=y_{1}, \lambda_{2}(y)=$ $y_{2}, \ldots, \lambda_{n}(y)=y_{n}$, fulfil conditions (4) and (5) and $d \lambda_{i}(0) \neq 0$. (If) The existence of a series of nested distributions that are involutive and nonsingular implies according to [19, Lemma 6.4] the existence of a set of coordinates $z=\Phi(x)$, in which the distributions are described by

$$
\Delta_{i}(z)=\operatorname{span}\left\{\frac{\partial}{\partial z_{1}}, \frac{\partial}{\partial z_{2}} \ldots, \frac{\partial}{\partial z_{i}}\right\} .
$$

${ }^{2}$ Without loss of generality, inthe rest of this note it is assumed that $x^{0}=0$. 
Next, consider $n$ functions $\lambda_{1}(z), \lambda_{2}(z), \ldots, \lambda_{n}(z)$ such that (4) and (5) hold. Because of the expression (6), condition (4) implies that each function $\lambda_{i}(z)$ will in fact be a function of $\left(z_{i}, z_{i+1}, \ldots, z_{n}\right)$ with $\left(\partial \lambda_{i} / \partial z_{i}\right)$ not identically equal to zero at zero, for all $i$. For that, and because $d \lambda_{i}(0) \neq 0$, the functions $\lambda_{1}(z), \lambda_{2}(z), \ldots, \lambda_{n}(z)$ define a local diffeomorphism

$$
y=\Lambda(z)=\left[\begin{array}{c}
\lambda_{1}\left(z_{1}, z_{2}, \ldots, z_{n-1}, z_{n}\right) \\
\lambda_{2}\left(z_{2}, \ldots, z_{n-1}, z_{n}\right) \\
\vdots \\
\lambda_{n-1}\left(z_{n-1}, z_{n}\right) \\
\lambda_{n}\left(z_{n}\right)
\end{array}\right] .
$$

We obtain

$$
\dot{y}=\left[\begin{array}{c}
L_{\tilde{f}} \lambda_{1}(z) \\
L_{\tilde{f}} \lambda_{2}(z) \\
\vdots \\
L_{\tilde{f}} \lambda_{n-1}(z) \\
L_{\tilde{f}} \lambda_{n}(z)
\end{array}\right]_{z=\Lambda^{-1}(y)}+\left[\begin{array}{c}
L_{\tilde{g}} \lambda_{1}(z) \\
L_{\tilde{g}} \lambda_{2}(z) \\
\vdots \\
L_{\tilde{g}} \lambda_{n-1}(z) \\
L_{\tilde{g}} \lambda_{n}(z)
\end{array}\right]_{z=\Lambda^{-1}(y)} u .
$$

Conditions (5), written in the $z$-coordinates, are

$$
\begin{aligned}
\frac{\partial}{\partial z_{1}} L_{\tilde{f}} \lambda_{1}(z) & =\frac{\partial}{\partial z_{1}} L_{\tilde{f}} \lambda_{2}(z)=\frac{\partial}{\partial z_{2}} L_{\tilde{f}} \lambda_{2}(z)=\cdots \\
& =\frac{\partial}{\partial z_{i}} L_{\tilde{f}} \lambda_{i}(z)=0 \\
\frac{\partial}{\partial z_{1}} L_{\tilde{g}} \lambda_{1}(z) & =\frac{\partial}{\partial z_{1}} L_{\tilde{g}} \lambda_{2}(z)=\frac{\partial}{\partial z_{1}} L_{\tilde{g}} \lambda_{2}(z)=\cdots \\
& =\frac{\partial}{\partial z_{i}} L_{\tilde{g}} \lambda_{i}(z)=0 .
\end{aligned}
$$

From (9), and from the triangular structure of the transformation (7), we conclude that the system is transformed into the form

$$
\dot{y}=\left[\begin{array}{c}
\hat{f}_{1}\left(y_{2}, \ldots, y_{n-1}, y_{n}\right) \\
\hat{f}_{2}\left(y_{3}, \ldots, y_{n-1}, y_{n}\right) \\
\vdots \\
\hat{f}_{n-1}\left(y_{n}\right) \\
0
\end{array}\right]+\left[\begin{array}{c}
\hat{g}_{1}\left(y_{2}, \ldots, y_{n-1}, y_{n}\right) \\
\hat{g}_{2}\left(y_{3}, \ldots, y_{n-1}, y_{n}\right) \\
\vdots \\
\hat{g}_{n-1}\left(y_{n}\right) \\
c_{g}
\end{array}\right] u
$$

Hence, the claim.

Remark 2: If a system is coordinates equivalent to a strict feedforward form, then it is necessarily coordinates equivalent to a feedforward form. Indeed conditions (3), e.g., the invariance of the distributions under the vector fields $f$ and $g$, are implied by the existence of the functions $\lambda_{1}(x), \lambda_{2}(x), \ldots, \lambda_{n}(x)$ with the properties (4) and (5). This can be easily verified considering the change of coordinates (7) and the (9), and keeping in mind that $\Delta_{i}(z)=\operatorname{span}\left\{\left(\partial / \partial z_{1}\right),\left(\partial / \partial z_{2}\right), \ldots,\left(\partial / \partial z_{i}\right)\right\}$ and $\Delta_{i}(y)=\operatorname{span}\left\{\left(\partial / \partial y_{1}\right),\left(\partial / \partial y_{2}\right), \ldots,\left(\partial / \partial y_{i}\right)\right\}$.

Remark 3: To establish coordinate equivalence to a strict feedforward form, one might be tempted to consider the conditions

$$
\left[f, \Delta_{i}\right] \subset \Delta_{i-1} \quad\left[g, \Delta_{i}\right] \subset \Delta_{i-1} .
$$

However, for a vector field $g$ satisfying condition (11) the following implications are true [as in the rest of the chapter, we denote with $\tilde{g}$ the vector field expressed in the coordinates in which the set of distributions $\Delta_{i}$ are expressed by (6)]. For all $i=1, \ldots, n$ and all scalar functions $\mu(z)$ the vector field $\mu(z)\left(\partial / \partial z_{i}\right)$ is in $\Delta_{i}$, whereas all vectors in $\Delta_{i-1}$ have zero entries in the positions $i, i+1, \ldots, n$. Then, by $(11)\left(\partial \mu / \partial z_{i}\right) \tilde{g}-\mu(z)\left(\partial \tilde{g} / \partial z_{i}\right) \subset \Delta_{i-1} \Rightarrow\left(\partial \mu / \partial z_{i}\right) \tilde{g}_{i}-$ $\mu(z)\left(\partial \tilde{g}_{i} / \partial z_{i}\right)=0$. However, this is true for all functions $\mu(z)$ only if $\tilde{g}_{i}=0$ for all $i=1, \ldots, n$, i.e., $\tilde{g}(z)=g(x)=0$. The same applies to the vector field $f$. Hence, conditions (11) are not correct.

\section{B. A Necessary Condition for Strict Feedforward Forms}

The applicability of the results in Propositions 1 and 2 depends on the solution of some nontrivial partial differential equations. On the other hand, it is known that a necessary and sufficient condition for a linear system to be coordinates equivalent to a system in strict feedforward form is that its eigenvalues are equal to zero. Derivation of similar, easy-to-check necessary conditions for nonlinear systems would prove very useful. In this section we present a necessary condition for the coordinates equivalence of a nonlinear system (1) to a system in strict feedforward form. For simplicity, we restrict ourselves to the first step of the procedure and consider a system with no controls, i.e., we are looking for equivalence to the form

$$
\dot{y}=\left[\begin{array}{c}
\hat{f}_{1}\left(y_{2}, \ldots, y_{n}\right) \\
\hat{f}_{2}\left(y_{2}, \ldots, y_{n}\right) \\
\vdots \\
\hat{f}_{n}\left(y_{2}, \ldots, y_{n}\right)
\end{array}\right] .
$$

Consider a nonzero vector field $\tau_{1}$ and the one-dimensional involutive distribution $\Delta_{1}=\operatorname{span}\left\{\tau_{1}\right\}$. According to Proposition 2 (see also the explanation in [9, Rem. 9.1]), for a system that is coordinate equivalent to a at-the-first-step strict feedforward form, i.e., to a system of the form (12), there exist a one-dimensional distribution and $n$ real functions $\lambda_{1}(x), \lambda_{2}(x), \ldots, \lambda_{n}(x)$ such that the following hold.

1) Conditions (4): $\Delta_{1}^{\perp} \not \nexists d \lambda_{1} \in I R^{n}$ and $d \lambda_{i} \in \Delta_{1}^{\perp}$, for $i=$ $2, \ldots, n$, i.e.,

$$
\left\langle d \lambda_{1}, \tau_{1}\right\rangle \neq 0 \quad \text { and } \quad\left\langle d \lambda_{i}, \tau_{1}\right\rangle=0, \quad i=2, \ldots, n .
$$

2) Condition (5): $d L_{f} \lambda_{i} \in \Delta_{1}^{\perp}$, i.e.,

$$
\left\langle d\left(\left\langle d \lambda_{i}, f\right\rangle\right), \tau_{1}\right\rangle=0 \Longleftrightarrow d \lambda_{i} \frac{\partial f}{\partial x} \tau_{1}+f^{\prime} \frac{\partial^{2} \lambda_{i}}{\partial x^{2}} \tau_{1}=0 .
$$

As mentioned in Remark 2, the distribution $\Delta_{1}$ is invariant under the vector field $f$ (and the same holds for $g$ ), i.e., there exists a real function $k(x)$ such that

$$
\left[f, \tau_{1}\right]=\frac{\partial \tau_{1}}{\partial x} f-\frac{\partial f}{\partial x} \tau_{1}=k(x) \tau_{1} .
$$

Multiplying both sides of (15) by the nonsingular matrix

$$
M=\left[\begin{array}{c}
d \lambda_{1} \\
\vdots \\
d \lambda_{n}
\end{array}\right]
$$

and using (13) and (14) we obtain

$$
\left[\begin{array}{c}
d \lambda_{1} \frac{\partial \tau_{1}}{\partial x} f \\
d \lambda_{2} \frac{\partial \tau_{1}}{\partial x} f \\
\vdots \\
d \lambda_{n} \frac{\partial \tau_{1}}{\partial x} f
\end{array}\right]+\left[\begin{array}{c}
f^{\prime} \frac{\partial^{2} \lambda_{1}}{\partial x^{2}} \tau_{1} \\
f^{\prime} \frac{\partial^{2} \lambda_{2}}{\partial x^{2}} \tau_{1} \\
\vdots \\
f^{\prime} \frac{\partial^{2} \lambda_{n}}{\partial x^{2}} \tau_{1}
\end{array}\right]=k(x)\left[\begin{array}{c}
d \lambda_{1} \tau_{1} \\
0 \\
\vdots \\
0
\end{array}\right]
$$

A necessary condition for coordinates equivalence to an at-thefirst-step strict feedforward form can be obtained from the first row of the matrix (16), which rewrites as $d \lambda_{1}\left(\partial \tau_{1} / \partial x\right) f+$ $\tau_{1}^{\prime}\left(\partial^{2} \lambda_{1} / \partial x^{2}\right) f=k(x) d \lambda_{1} \tau_{1}$, i.e.,

$$
\frac{\partial}{\partial x}\left(L_{\tau_{1}} \lambda_{1}\right) f=k(x) L_{\tau_{1}} \lambda_{1}
$$

Moreover, by (13) $L_{\tau_{1}} \lambda_{1} \neq 0$, hence $k(0)=0$. A necessary condition can be obtained by conditions (15) and (17). 
Corollary 1: If a vector field $f(x)$ is coordinates equivalent to a vector field which is at-the-first-step in a strict feedforward form, then there exist a vector field $\tau_{1} \neq 0$ and functions $\lambda_{1}(x)$ and $k(x)$ with $d \lambda_{1} \neq 0$ and $k(0)=0$ such that (15) and (17) hold.

An immediate consequence of Corollary 1 is that if a linear system $\dot{x}=A x$ is coordinates equivalent to a system which is at-the-first-step in a strict feedforward form, then the determinant of $A$ is equal to zero.

\section{Feedback Equivalence}

In this section we show how the conditions expressed in Propositions 1 and 2 have to be modified in order to solve the feedback equivalence problem, i.e., Problem 2. By [6, Lemma 6.2.1], and the results in [19, Lemma 6.4] and [19, Th. 3.49] or [6, Lemma 1.6.1] the following can be established.

Proposition 3: Consider system (1). Let distributions (2) be such that $\Delta_{i}$ is $i$-dimensional and involutive, and such that the distributions $\Delta_{i}+\operatorname{span}\{g(x)\}$ are nonsingular. Then the system (1) is locally feedback equivalent to a system in feedforward form if and only if

$$
\left[f, \Delta_{i}\right] \subset \operatorname{span}\{g\}+\Delta_{i} \quad\left[g, \Delta_{i}\right] \subset \operatorname{span}\{g\}+\Delta_{i}
$$

for all $i=1, \ldots, n-1$.

Remark 4: It is worth noting that the results concerning the feedback equivalence are not as elegant as the ones on coordinates equivalence. This is because in the proof of the sufficiency of the feedback equivalence we need the technical assumption that the distributions $\Delta_{i}+\operatorname{span}\{g(x)\}$ be nonsingular. This assumption is required to construct the feedback laws inducing the invariance of the distributions $\Delta_{i}$ (in the closed loop system), but it is not necessary. As a matter of fact the regularity of the above distributions is not needed in the proof of the necessity. This fact should not be surprising and is a consequence of the gap existing in the characterization of locally controlled invariant distributions, as observed in [6, Rem. 6.2.1]. To address the problem of feedback equivalence to strict feedforward forms, we present first the following preliminary result.

Proposition 4: System (1) is locally feedback equivalent to a system in strict feedforward form if and only if there exists a coordinates transformation $z=\Phi(x)$ such that (1) written in the $z$-coordinates is described by equations of the form

$$
\dot{z}=b(z)(c(z)+e(z) u)+h(z)
$$

where $c(z)$ and $e(z)$ are scalar functions, with $e(0) \neq 0$, and $b(z)$ and $h(z)$ are vector fields in strict feedforward form, i.e.,

$$
b(z)=\left[\begin{array}{c}
b_{1}\left(z_{2}, \ldots, z_{n}\right) \\
b_{2}\left(z_{3}, \ldots, z_{n}\right) \\
\vdots \\
c_{d}
\end{array}\right] \quad h(z)=\left[\begin{array}{c}
h_{1}\left(z_{2}, \ldots, z_{n}\right) \\
h_{2}\left(z_{3}, \ldots, z_{n}\right) \\
\vdots \\
c_{h}
\end{array}\right] .
$$

Proof: (Only If) Suppose that system (1) is locally feedback equivalent to a system in strict feedforward form. Then, there exists a feedback $u(x)=\alpha(x)+\beta(x) v$, with $\beta(x) \neq 0$ and a coordinates transformation $z=\Phi(x)$ such that the system $\dot{z}=\tilde{f}_{f}(z)+\tilde{g}_{f}(z) v$ is in strict feedforward form, with $f_{f}(x)=f(x)+g(x) \alpha(x), g_{f}(x)=g(x) \beta(x), \tilde{f}_{f}(z)=$ $\left.(\partial \Phi / \partial x) f_{f}(x)\right|_{x=\Phi^{-1}(z)}$ and $\tilde{g}_{f}(z)=\left.(\partial \Phi / \partial x) g_{f}(x)\right|_{x=\Phi^{-1}(z)}$. Applying the transformation $z=\Phi(x)$ to the original system (1), one gets

$$
\dot{z}=\tilde{f}(z)+\tilde{g}(z) u
$$

which is necessarily transformed into a strict feedforward form by the feedback $u(z)=\left.u(x)\right|_{x=\Phi-1(z)}=\tilde{\alpha}(z)+\tilde{\beta}(z) v$. This implies that (see [9] for details) the vector fields $\tilde{g}(z)$ and $\tilde{f}(z)$ must possess a special structure, namely

$$
\tilde{g}(z)=\psi_{g}(z) \tilde{g}_{n}(z) \quad \text { and } \quad \tilde{f}(z)=\psi_{f}(z)+\psi_{g}(z) \tilde{f}_{n}(z)
$$

where $\psi_{g}(z)$ and $\psi_{f}(z)$ are vector fields in strict feedforward form. From (21), we conclude that system (20) can be written in the form of (19) with $c(z)=\tilde{f}_{n}(z), e(z)=\tilde{g}_{n}(z), b(z)=\psi_{g}(z)$ and $h(z)=$ $\psi_{f}(z)$, i.e., the vector fields $\tilde{f}(z)$ and $\tilde{g}(z)$ are described by $\tilde{f}(z)=$ $h(z)+b(z) \tilde{f}_{n}(z)$ and $\tilde{g}(z)=b(z) \tilde{g}_{n}(z)$, which proves the claim.

(If) Consider a system described by (19). Applying the feedback $u(z, v)=-(c(z) / e(z))+(1 / e(z)) v$ we get $\dot{z}=h(z)+b(z) v$ which is in strict feedforward form and feedback equivalent to (1).

Proposition 4 gives necessary and sufficient conditions for feedback equivalence to strict feedforward forms, however, these conditions are not phrased in the geometric framework that has been used for the results presented thus far. We overcome this shortcoming with the next result.

Proposition 5: Consider system (1). Let distributions (2) be such that $\Delta_{i}$ are $i$-dimensional and involutive and such that the distributions $\Delta_{i}+\operatorname{span}\{g(x)\}$ are nonsingular. Then the system (1) is locally feedback equivalent to a system in a strict feedforward form, if and only if

- $\quad$ there exist $n$ real functions $\lambda_{1}(x), \lambda_{2}(x), \ldots, \lambda_{n}(x)$ with $d \lambda_{i}(0) \neq 0$, such that (4) holds, $L_{g} \lambda_{n} \neq 0$ and, moreover

$$
\begin{gathered}
d L_{f} \lambda_{1}-\frac{L_{g} \lambda_{1}}{L_{g} \lambda_{n}} d L_{f} \lambda_{n} \in \Delta_{1}^{\perp} \\
d L_{f} \lambda_{2}-\frac{L_{g} \lambda_{2}}{L_{g} \lambda_{n}} d L_{f} \lambda_{n} \in \Delta_{2}^{\perp} \\
\vdots \\
d L_{f} \lambda_{n-1}-\frac{L_{g} \lambda_{n-1}}{L_{g} \lambda_{n}} d L_{f} \lambda_{n} \in \Delta_{n-1}^{\perp} \\
d L_{g} \lambda_{1}-\frac{L_{g} \lambda_{1}}{L_{g} \lambda_{n}} d L_{g} \lambda_{n} \in \Delta_{1}^{\perp} \\
d L_{g} \lambda_{2}-\frac{L_{g} \lambda_{2}}{L_{g} \lambda_{n}} d L_{g} \lambda_{n} \in \Delta_{2}^{\perp} \\
\vdots \\
d L_{g} \lambda_{n-1}-\frac{L_{g} \lambda_{n-1}}{L_{g} \lambda_{n}} d L_{g} \lambda_{n} \in \Delta_{n-1}^{\perp}
\end{gathered}
$$

Remark 5: A system that is locally feedback equivalent to a strict feedforward form is locally feedback equivalent to a feedforward form. Note, in fact, that conditions (18), i.e., the necessary and sufficient conditions for the controlled invariance of the distributions $\Delta_{i}$, can be recovered from conditions (22).

Proof: (Only If) Assuming that system (1) is locally feedback equivalent to a system in strict feedforward form and following the steps in the proof of Proposition 4 we see that conditions (22) are satisfied for the distributions $\Delta_{i}=\operatorname{span}\left\{\left(\partial / \partial z_{i}\right)\right\}, i=1, \ldots, n-1$ and the functions $\lambda_{i}=z_{i}$.

(If) Consider system (1) and the change of coordinates $z=\Lambda(x)=$ $\operatorname{col}\left[\lambda_{1}(x), \lambda_{2}(x), \ldots, \lambda_{n}(x)\right]$, where the functions $\lambda_{i}$ are such that condition (4) holds, with the nested sequence of distributions of (2). This gives the following equivalent representation of (1):

$$
\dot{z}=\left[\begin{array}{c}
L_{f} \lambda_{1}(x) \\
L_{f} \lambda_{2}(x) \\
\vdots \\
L_{f} \lambda_{n}(x)
\end{array}\right]_{x=\Lambda^{-1}(z)}+\left[\begin{array}{c}
L_{g} \lambda_{1}(x) \\
L_{g} \lambda_{2}(x) \\
\vdots \\
L_{g} \lambda_{n}(x)
\end{array}\right]_{x=\Lambda^{-1}(z)} u .
$$


Next, since it is assumed that $L_{g} \lambda_{n} \neq 0$, we define the feedback $u=-\left(L_{f} \lambda_{n} / L_{g} \lambda_{n}\right)+\left(1 / L_{g} \lambda_{n}\right) v$. Using this feedback, system (23) becomes

$$
\dot{z}=\left[\begin{array}{c}
L_{f} \lambda_{1}-\frac{L_{f} \lambda_{n}}{L_{g} \lambda_{n}} L_{g} \lambda_{1} \\
L_{f} \lambda_{2}-\frac{L_{f} \lambda_{n}}{L_{g} \lambda_{n}} L_{g} \lambda_{2} \\
\vdots \\
0
\end{array}\right]+\left[\begin{array}{c}
\frac{L_{g} \lambda_{1}}{L_{g} \lambda_{n}} \\
\frac{L_{g} \lambda_{2}}{L_{g} \lambda_{n}} \\
\vdots \\
1
\end{array}\right] v \triangleq f^{\star}+g^{\star} v .
$$

Using (22), we notice that for $i=1, \ldots, n-1$

$$
d g_{i}^{\star}=\frac{L_{g} \lambda_{n} d L_{g} \lambda_{i}-L_{g} \lambda_{i} d L_{g} \lambda_{n}}{\left(L_{g} \lambda_{n}\right)^{2}} \in \Delta_{i}^{\perp} .
$$

For the vector field $f^{\star}$, keeping in mind that $g_{i}^{\star}=\left(L_{g} \lambda_{i} / L_{g} \lambda_{n}\right)$ and that $d g_{i}^{\star} \in \Delta_{i}^{\perp}$, we have

$$
d f_{i}^{\star}=d\left(L_{f} \lambda_{i}-g_{i}^{\star} L_{f} \lambda_{n}\right) \in \Delta_{i}^{\perp} .
$$

Then, from Proposition 2 it follows that the vector fields $f^{\star}$ and $g^{\star}$ are coordinates equivalent to a strict feedforward form.

\section{AN EXAMPLE}

Consider a four-dimensional model of a food-chain system [5], [20], i.e.,

$$
\begin{aligned}
& \dot{x}_{1}=-x_{1}+x_{1} x_{2} \\
& \dot{x}_{2}=-x_{1} x_{2}-x_{2}+x_{2} x_{3} \\
& \dot{x}_{3}=-x_{2} x_{3}-x_{3}+x_{3} x_{4} \\
& \dot{x}_{4}=-x_{3} x_{4}-x_{4}+u .
\end{aligned}
$$

This system describes the behavior of a (normalized) four species ecology, in which the species described by $x_{2}$ and $x_{3}$ act as preys and predators, $x_{1}$ acts as predator and $x_{4}$ acts as prey. The species described by $x_{4}$ is fed by the environment through the input signal $u$. Obviously, the system is defined in the (open) positive orthant $I R_{+}^{4}$, which is a positive invariant set for all trajectories as long as $u>0$. The qualitative behavior of system (24) has been extensively studied in the biological and game theory communities, whereas a few control problems have been discussed in [20]. System (24) is not in feedforward form, and there is no obvious change of feedback and/or change of coordinates that transforms the system into a feedforward form. Nevertheless, the system can be (locally) transformed into a feedforward form, as detailed in the following statement.

Proposition 6: System (24) is locally feedback equivalent to a feedforward form around any point $x_{0} \in I R_{+}^{4}$.

Proof: Simple but tedious computations show that the distribution

$$
\Delta_{1}=\operatorname{span}\left\{\left[\begin{array}{c}
x_{3} \\
0 \\
x_{3} \\
2 x_{2}-x_{4}
\end{array}\right]\right\}
$$

is a controlled invariant distributions for system (24). Hence, following the construction in [6, Lemma 1.6.1], we define (locally) new coordinates $z_{1}=\phi_{1}(x), z_{2}=\phi_{2}(x), z_{3}=\phi_{3}(x)$ and $z_{4}=\phi_{4}(x)$ such that $\operatorname{span}\left\{d \phi_{2}, d \phi_{3}, d \phi_{4}\right\}=\Delta_{1}^{\perp}$. A possible choice for such new coordinates is

$$
z=\left[\begin{array}{c}
z_{1} \\
z_{2} \\
z_{3} \\
z_{4}
\end{array}\right]=\left[\begin{array}{c}
x_{1} \\
x_{2} \\
x_{3}-x_{2}-x_{1} \\
x_{3}\left(x_{4}-2 x_{2}\right)
\end{array}\right]
$$

The aforementioned coordinates transformation is a diffeomorphism on $I R_{+}^{4}$. In the new coordinates, (24) is described by the equations

$$
\begin{aligned}
\dot{z}_{1}= & -z_{1}+z_{1} z_{2} \\
\dot{z}_{2}= & -z_{2}+z_{2}^{2}+z_{2} z_{3} \\
\dot{z}_{3}= & -z_{3}+z_{4} \\
\dot{z}_{4}= & \frac{z_{4}^{2}}{z_{1}+z_{2}+z_{3}}-4 z_{2}\left(z_{1}+z_{2}+z_{3}\right)^{2} \\
& +z_{2} z_{4}-2 z_{4}+\left(u+2 z_{1} z_{2}-z_{4}\right)\left(z_{1}+z_{2}+z_{3}\right) .
\end{aligned}
$$

Hence, after a proper redefinition of the control $u$, the conclusion follows.

Remark 6: Though the coordinate transformation proposed previously is not defined for $x_{3}=0$, the result holds in the open positive orthant. As the system represents a food chain, and it is a positive system, it is itself defined only in the positive orthant thus the limitation of the design of the transformation is not restrictive.

\section{CONCLUSION AND OUTLOOK}

The problems of local coordinates and feedback equivalence of a single-input nonlinear system to a class of feedforward systems have been studied and a geometric characterization of the problems has been proposed. As the definition of feedforward systems is not unique, we restrict out investigation to a special subclass. However, similar conditions can be given for more general classes of systems and in particular for the so-called block feedforward systems. The main results of the note are necessary and sufficient conditions that are phrased via the existence of some invariant and controlled invariant distributions. The implementation requires the solution of an intricate partial differential equations problem.

The global problem and the case of multiple-input systems are still open and are more complicated than the one considered. In particular, to obtain global statements it is necessary to require existence of global (controlled) invariant distributions and completeness of the vector fields in the distributions, whereas no major differences are to be expected in dealing with multiple-input systems. We leave these problems open for further investigation.

\section{REFERENCES}

[1] E. Aranda-Bricaire and C. H. Moog, "Equivalence of discrete-time nonlinear systems to feedforward form," presented at the Eur. Control Conf., Cambridge, MA, Sep. 1-4, 2003.

[2] - "Invariant codistributions and the feedforward form for discretetime nonlinear systems," Syst. Control Lett., vol. 52, pp. 113-122, 2004.

[3] A. Astolfi and F. Mazenc, "A geometric characterization of feedforward forms," presented at the MTNS'2000, Perpignan, France, 2000.

[4] S. Battilotti, "Semiglobal stabilization of uncertain block-feedforward systems via measurement feedback," presented at the IFAC Nonlinear Control Systems Design Symp., Enschede, The Netherlands, 1998.

[5] J. Hofbaner and K. Sigmund, Evolutionary Games and Population Dynamics. Cambridge, U.K.: Cambridge Univ. Press, 1998.

[6] A. Isidori, Nonlinear Control Systems, 3rd ed. New York: SpringerVerlag, 1995

[7] — Nonlinear Control Systems II. New York: Springer-Verlag, 1999.

[8] M. Janković, R. Sepulchre, and P. V. Kokotović, "Constructive lyapunov stabilization of nonlinear cascade systems," IEEE Trans. Autom. Control, vol. 41, no. 12, pp. 1723-1735, Dec. 1996.

[9] G. Kaliora, "Control of nonlinear systems with bounded signals," Ph.D. dissertation, Imperial College, Univ. London, London, U.K., 2002. 
[10] G. Kaliora and A. Astolfi, "Nonlinear control of feedforward systems with bounded signals," IEEE Trans. Autom. Control, vol. 49, no. 11, pp. 1975-1990, Nov. 2004.

[11] W. Kang, "Extended controller form and invariants of nonlinear control systems with a single input," J. Math. Syst., Estimat., Control, vol. 6, pp. 27-51, 1996.

[12] W. Kang and A. J. Krener, "Extended quadratic controller normal form and dynamic state feedback linearization of nonlinear systems," SIAM J. Control Optim., vol. 30, no. 6, pp. 1319-1337, Nov. 1992.

[13] V. Kozlov, Symmetries, Topology and Resonances in Hamiltonian Mechanics. New York: Springer-Verlag, 1996.

[14] W. Lin and X. Li, "Synthesis of upper-triangular nonlinear systems with marginally unstable free dynamics using state dependent saturation," Int. J. Control, vol. 72, pp. 1078-1086, 1999.

[15] W. Lin and C. Qian, "New results on global stabilization of feedforward systems via small control," in Proc. 37th IEEE Conf. Decision and Control, 1998, pp. 873-878.

[16] L. Marconi and A. Isidori, "Robust global stabilization of a class of uncertain feedforward nonlinear systems," Syst. Control Lett., vol. 41, no. 4, pp. 281-290, 2000.

[17] F. Mazenc and L. Praly, "Adding integrations, saturated controls and stabilization for feedforward systems," IEEE Trans. Autom. Control, vol. 41, no. 11, pp. 1559-1578, Nov. 1996.

[18] F. Mazenc, R. Sepulchre, and M. Jankovic, "Lyapunov functions for stable cascades and applications to global stabilization," IEEE Trans. Autom. Control, vol. 44, no. 9, pp. 1795-1800, Sep. 1999.

[19] H. Nijmeijer and A. J. Van der Schaft, Nonlinear Dynamical Control Systems. New York: Springer-Verlag, 1989.

[20] R. Ortega, A. Astolfi, G. Bastin, and H. Rodriguez-Cortes, "Output feedback control of food chain systems," in New Trends in Nonlinear Observer Design, H. Nijmeijer and T. Fossen, Eds. New York: SpringerVerlag, 1999, pp. 291-310.

[21] W. Respondek, "Transforming nonholonomic control systems into the canonical contact form," in Proc. 40th Conf. Decision and Control, Orlando, FL, 2001, pp. 1781-1786.

[22] R. Sepulchre, M. Jankovic, and P. V. Kokotović, Constructive Nonlinear Control. New York: Springer-Verlag, 1996.

[23] I. A. Tall and W. Respondek, "Transforming a single-input nonlinear system to a strict feedforward form via feedback," in Nonlinear Control in the Year 2000, A. Isidori, F. Lamnabhi-Lagarrigue, and W. Respondek, Eds. London, U.K.: Springer-Verlag, 2000, vol. 2, pp. 527-542.

[24] A. Teel, "Feedback stabilization: Nonlinear solutions to inherently nonlinear problems," Dept. Comput. Sci., Univ. California, Berkeley, CA, Memorandum ERL M92/65, 1992.

\section{Simultaneous Observation of Linear Systems: A State-Space Interpretation}

\author{
Jaime A. Moreno
}

\begin{abstract}
Necessary and sufficient conditions for the existence of a simultaneous functional observer for several linear time-invariant (LTI) systems are derived using distinguishability concepts, that generalize the detectability concept for a single system. They generalize also the known results. A structure for common functional observers is proposed, and for two systems, or for several systems without inputs, the problem can be constructively solved. A relationship between the simultaneous observation problem and the existence of unknown input observers is established.
\end{abstract}

Index Terms-Functional observers, indistinguishability, input decoupled observers, linear observers, robust observation, simultaneous observation, unknown input observers.

\section{INTRODUCTION}

The problem of designing a single observer for two or several linear plants, i.e., the so called simultaneous observation problem, is of interest when, for example, a robust observer has to be designed that converges despite of failures in some components of a system. Or when for a nonlinear system, that has several operating points, an (linear) observer has to be designed. In contrast to the (dual) simultaneous control problem [12], that has received a lot of attention in the literature since its appearance around 1980, the simultaneous observation problem has only recently been studied [6], [13]. In these references, the problem has been characterized using matrix fraction description (MFD).

The objective of this note is to give an state-space characterization of the solvability conditions for the problem. In doing so the results in [13] are generalized to include not detectable and/or not stabilizable systems, and systems without inputs. Moreover, a general structure for simultaneous observers is found. A second objective is also to obtain an "intuitive" characterization of the conditions for simultaneous functional observability in system theoretic terms, i.e., in terms of trajectory behavior. It is shown that simultaneous observation of two systems is equivalent to an easy to grasp detectability condition, that generalizes detectability for a single system. Moreover, it is shown that the simultaneous observation problem is intimately related to the problem of unknown input observation, a classical observation problem. In fact, the simultaneous observation of $N$ systems is reduced to the problem of constructing a simultaneous observer for a family of $N-1$ systems, but with unknown inputs. This shows that for $N>2$ a complete characterization of our problem requires the solution of the same problem for systems with unknown inputs.

The note is organized as follows. In Section II, some preliminary results are reviewed, and the problem solved in this note is stated. The simultaneous observation problem for two systems is completely solved in Section III, and in Section IV a necessary and sufficient condition is obtained for a finite number of systems.

Let $\lambda(A)$ be the set of eigenvalues of $A, \mathbb{C}^{-}$and $\mathbb{C}_{0}^{+}$the open left and the closed right half planes of the complex plane, respectively. $\lim _{t \rightarrow \infty} z(t)=\lim _{t \rightarrow \infty} g(t)$ will be represented as $z(t) \rightarrow g(t)$.

Manuscript received March 27, 2003; revised February 15, 2004 and October 28, 2004. Recommended by Associate Editor L. E. Holloway. This work was supported by CONACyT under Project 34934A, and by DGAPA-UNAM under Project PAPIIT IN111905.

The author is with Instituto de Ingeniería, Universidad Nacional Autónoma de México, Ciudad Universitaria, 04510 Coyoacán, México City, Mexico (e-mail: JMorenoP@iingen.unam.mx).

Digital Object Identifier 10.1109/TAC.2005.851447 